\title{
A Brief Review of Firefly Algorithm: Application in Structural Optimization Problem
}

\author{
Sujan Tripathi \\ Department of Civil Engineering, Thapathali Campus \\ Institute of Engineering, Tribhuvan University, Kathmandu, Nepal \\ Corresponding author: sujantripathi@ioe.edu.np
}

Received: Dec 21, 2018

Revised: Feb 15, 2019

Accepted: Feb 18, 2019

\begin{abstract}
Firefly Algorithm is a recently developed meta-heuristic algorithm, which is inspired by the flashing behaviour of Firefly. Initially, Firefly algorithm was used to solve the optimization problems of continuous search domain. Further, many researchers have successfully implemented this algorithm in several discrete optimization problems. Although the firefly algorithm behaves like another meta-heuristic method (i.e. Particle Swarm Optimization particle), however, the firefly is robust than that. Due to the presence of an exponential term in its movement equation, firefly algorithm is capable to search optimum value more efficiently than others. This study is, mainly, focused to show the strength of the firefly algorithm to solve the complex problems and to explore the possible research area on the structural engineering field. This study shows about the robustness of the firefly algorithm on the basis of recently published papers that was used to solve the size, shape and topology optimization of the spatial truss structure with discrete design variables. The review result shows that the performance of the Firefly Algorithm is remarkable compared to other nature-inspired-algorithms, such as particle swarm optimization. This study concludes with some remarkable points that will be more beneficial to the future researchers of this area.
\end{abstract}

Keywords: Firefly algorithm, improved Firefly algorithm, hybridization, modification, truss optimization

\section{Introduction}

Firefly algorithm is one of a recent swarm intelligence method developed by Xin-She Yang in 2008. It is a stochastic, nature-inspired, meta-heuristic algorithm that can be applied to solve the hardest optimization problems. Stochastic means to use the randomization process for searching the set of solutions in a search domain. The stochastic method includes two branches called, heuristic and metaheuristic. Heuristic means 'to find' or 'to discover by trial and error [31]. It means there is no guarantee that the optimal solution will be found in a reasonable amount of time. Meta-heuristic means 'higher level', where the search process used in algorithms is influenced by a certain tradeoff between randomization and local search [30].

In the Firefly algorithm, the 'lower level' (heuristic) concentrates on the generation of new solutions within a search space and thus, selects the best solution for survival. On the other hand, 
randomization enables the search process to avoid the solution being trapped into local optima. The local search improves a candidate solution until improvements are detected. Metaheuristic is generally better than the heuristic algorithm since the search process is randomization and local search [31]. Even though metaheuristic only able to provide an "acceptable" solution, but it is not able to face complex and harder problems in a significant time [27]. Metaheuristic consists of two components: exploration (or diversification) and exploitation (or intensification) [6,31]. Exploration is the process of exploring the global search space thoroughly to discover the diverse solutions within that space. On the other hand, exploitation means the process of exploiting the local information to accelerate the search process and finally, helps to generate good solutions so far. Diversification is concerned with global search; therefore, it will increase the chance to gain the global optimality. But it will make the process slower with less convergence rate, whereas, intensification is about local search, which helps to converge the solution quickly. But again, there will be a chance of premature convergence and missing the global optima [35]. It is better to provide tread-off between these two components [31]. Balancing between these two components will provide the chance to diversify the search to reach nearer to the diversified solutions in a short time with the more chance to obtain the global optimality [6,35].

This paper covers a brief review of swarm intelligence based meta-heuristic algorithm, to show that it could be applicable to solve the complex structural engineering problems. On the other hand, it motivates and encourages the researchers who have an interest in solving structural design and optimization problems by using intelligence tools. Several kinds of literature are selected in this study. These are selected on the basis of literature that studied the real-field problem and demonstrates the remarkable outcomes.

The rest of the paper is outlined as follows: Section 2 presents the concept of firefly algorithm. Section 3 provides a clear description of firefly algorithm variants. Section 4 deals about the significance of algorithm in several engineering fields and Section 5 summarizes the paper with conclusion of the study.

\section{Standard Firefly Algorithm}

Firefly algorithm is a very recent meta-heuristic optimization algorithm developed by Yang [30, 31] and it is inspired by the flashing behaviour of fireflies. The movement of each Firefly is guided by its flashing pattern and attraction strategy. Three idealized rules are given below:

a. All fireflies are unisex so that one Firefly is attracted to other fireflies regardless of their sex.

b. Attractiveness is proportional to brightness, so for any two flashing fireflies, the less bright Firefly will move towards the brighter Firefly. Both attractiveness and brightness decrease as the distance between fireflies increases. If there is no Firefly brighter than a particular Firefly, that Firefly will move randomly.

c. The brightness of a Firefly is affected or determined by the landscape of the objective function.

The light intensity is decreased as the distance increase. It is also influenced by absorbed by the surrounding air, thus the intensity becomes less appealing as the distance increase [31]. The attractiveness of the fireflies is proportional to the light intensity seen by the other fireflies. 
Mathematically, $\quad \beta=\beta_{0} e^{-\gamma r_{i j}^{2}}$

where, $\beta_{0}$ is the attractiveness at $\boldsymbol{r}=\boldsymbol{0}$.

The distance between two fireflies can be defined by using Cartesian distance:

$$
\boldsymbol{r}_{i j}=\left\|\boldsymbol{x}_{\boldsymbol{i}}-\boldsymbol{x}_{\boldsymbol{j}}\right\|=\sqrt{\sum_{k=1}^{k=d}\left(x_{i, k}-x_{j, k}\right)^{2}}
$$

The less bright Firefly ' $i$ ' move towards the brighter Firefly ' $j$ '. Then, the movement equation is,

$$
\boldsymbol{X}_{i}^{t+1}=\boldsymbol{X}_{i}^{t}+\Delta \boldsymbol{X}_{i}^{t}=\boldsymbol{X}_{i}^{t}+\boldsymbol{\beta}\left(\boldsymbol{X}_{j}^{t}-\boldsymbol{X}_{i}^{t}\right)+\boldsymbol{\alpha} \boldsymbol{\epsilon}_{i}^{t} \quad\left[\because \beta=\beta_{0} e^{-\gamma r_{i j}^{2}}\right]
$$

In equation (3), first term representation of the initial position of Firefly. In the second term, $\beta$ represents the attractiveness which depends on brightness. ' $\gamma$ ' represents the absorption coefficient with the limiting values between 0 to $\infty$. If $\gamma \rightarrow 0$, then it indicates a Firefly can be seen anywhere in the space and easy to complete global search. If $\gamma \rightarrow \infty$, the attractiveness, and brightness decreases. Therefore, Firefly movements become random. The third term is for randomization, where ' $\alpha$ ' the randomize parameter and $\epsilon_{i}^{t}$ is a randomization vector. The letter ' $t$ ' represents the generation number counter during the process.

Pseudo code for standard Firefly algorithm is given in Fig. 1. It gives the clear picture of the Firefly algorithm and also describes that how the Firefly algorithm works

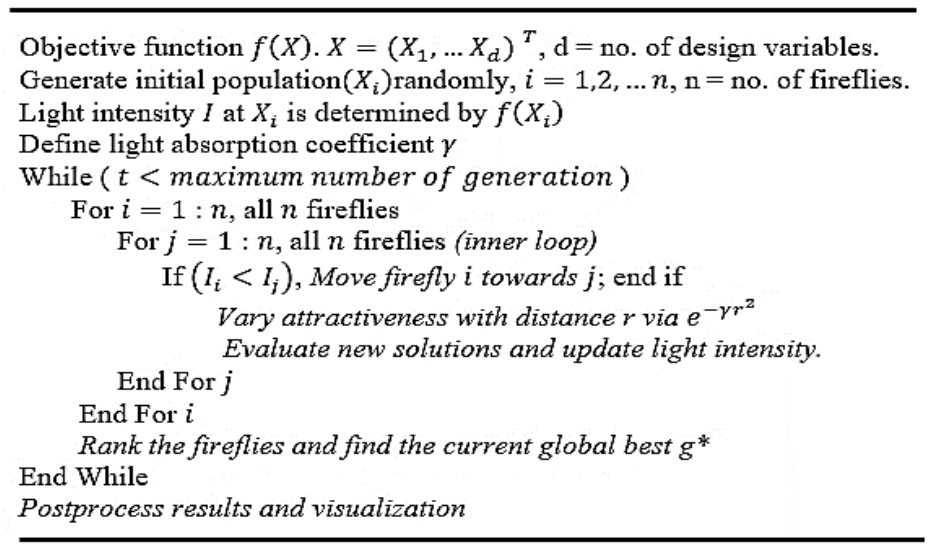

Fig. 1: Pseudo code for Standard Firefly algorithm [30]

\section{Variants of Firefly Algorithm}

At the beginning of its development, the classical firefly algorithm has been used mainly on continuous optimization problems. Classical firefly algorithm is not sufficient to get the desired solution if the size, variables, and complexity of problems are increased. Hence, the variants are necessary. Variants may be either in terms of a modification or in terms of hybridization to the classical firefly algorithm. In total, there are more than 20 different firefly algorithm variants [15].

\subsection{Modification of FA}

Although the classical firefly algorithm is a powerful method to optimize the truss, it suffers from the slow rate of convergence. Therefore, modification needs to improve the solution quality with 
reasonable convergence speed. Accelerated firefly algorithm improves the searching mechanism by using two strategies, i.e. gradual reduction of randomness and scaling the random term in fireflies' motion [3].

A classical firefly algorithm has some deficiencies in optimizing problems with large solution space, such as performing at a slow speed and high risk to trap on a poor local optimum. It will occur due to the wide distribution of initial individuals in the large solution space. To recover these deficiencies, a new firefly algorithm variant, named IFA, has proposed. It uses two strategies. First, embedding the random weight in classical firefly algorithm, that helps to enhance global exploration in the initial stage of the search process. Second, using improved attractiveness to enhance the local exploration that helps to provide the ability to escape from poor local optima in an accurate and fast way, and also helps to avoid excessive pace for location updating and lead to repeated oscillation in the last stage of the search process where the algorithm depends mainly on the local search $[25,29]$.

\subsection{Hybrid Firefly Algorithm}

A new hybrid metaheuristic approach by hybridizing harmony search (HS) with firefly algorithm (FA) (named as, HS/FA), is proposed to solve function optimization. Because of using the exploration of HS and the exploitation of FA, HS/FA has a faster convergence speed than HS and FA [18]. Farahani et al. [13] have published a paper with the use of a Genetic Algorithm (GA) hybridizing with Firefly Algorithm.

\section{Application of Firefly Algorithm in Structural Engineering}

FA is used by many researchers to solve the various type of problems such as routing problem [22], image processing [20], classification/clustering problems [26], optimization problems [3, 25, 29, $33,5,23,32,21,13,1]$. FA is also used to solve various structural engineering problems. It has successfully tested in the various field of structural engineering, such as, earthquake engineering [14], Beam section design [10,7] and design and optimization of steel structures [8], Shape, size and topology optimization of truss structures [3, 25, 29], Structural damage detection [12] etc.

\subsection{Firefly Algorithm in Structural Optimization}

In recent year, most of the researches are focused to solve the structural optimization problems because of the robustness of firefly algorithm to solve the complex problem. Several types of structural optimization problems are existing in the real world. They depend on objective functions selected, variables used, constraint types and the degree of complexity. Structural optimization problems are classified as; continuous [3], discrete [25, 29], combinatorial, constrained [17, 8], unconstrained [19], mixed variable [16], multi-objective [34], multi-modal [24], Multiple load case [3], dynamic [28, 2] and noisy [9].

\subsubsection{Firefly Algorithm in Truss Optimization}

The truss optimization problem is a type of complex structural problems. It consists of several variables, constraints, loading types with the objective function to minimize structural weight. Cost of truss structure is mainly depending on the weight of truss [4]. Minimum weight is generally taken as an objective function. Mainly, stress, displacement, stability, buckling, and natural frequency are taken as a constraint in truss optimization problems. There is three part of truss 
topology optimization. They are size optimization, shape optimization, and topology optimization. In size optimization, sectional area is taken as a variable. Shape optimization uses the coordinates of nodes as a variable. Similarly, connectivity is used as a variable in topology optimization. Several researchers carried their research on truss optimization because of its popularity in the construction field. In this study, only some recent literatures containing remarkable results are selected. Basically, this study aims to describe the robustness of firefly algorithm, to explorer the future research area and to show its scope in the structural engineering field. The selected literature is $[3,29]$.

\subsubsection{Review of Literature Results}

In this Section, the latest literature, published by Wu et al. [29] and Baghlani et al. [3] have selected to demonstrate the robustness and significance of the firefly algorithm and the modified firefly algorithm for complex engineering problems. These literatures are focused on size, shape and topology optimizations of trusses to incorporate the practical issues. Both of these papers have focused to solve the similar problems with discrete design variables as well as the similar load case. The first paper has introduced an improved strategy, named as IFA (improved firefly algorithm), to increase the performance and solution quality of classical firefly algorithm. And similarly, the second paper has introduced a new approach, named AFA (accelerated firefly algorithm), to speed up the solution convergence. Two spatial truss problems have taken on this study, which is shown in Fig. 2 and Fig. 3. The Fig. 2(a) and 2(b) show the initial geometry and the best topology with the minimum weight of 25-bars-spatial-truss, respectively. Similarly, Fig. 3(a), 3(b) and 3(c) show the initial geometry of 72-bars-spatial-truss and Fig. 3(d) is the final best topology of this truss with the minimum weight. The comparative study is carried out in this study to show the power, effectiveness, efficiency, and significance of the firefly algorithm to solve complex structural engineering problems. The dimensions used in Fig. 2(a) are in centimeter. The results are summarized in table 1 and 2.

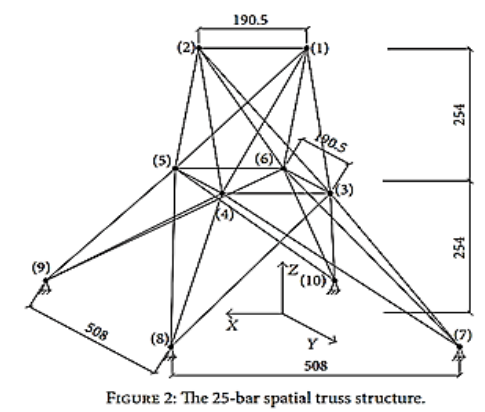

Fig. 2(a): 25-bar spatial truss

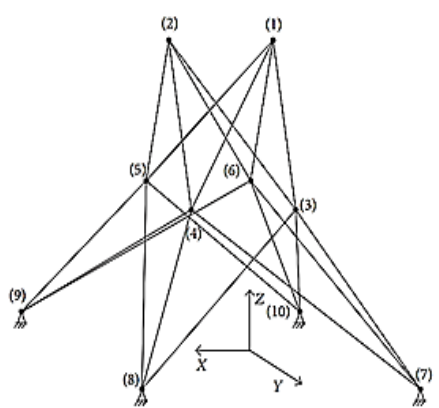

Fig. 2(b): Best topology of 25-bar spatial truss

Table 1: 25-bar spatial truss structure [29]

\begin{tabular}{|c|c|c|c|c|c|}
\hline \multirow{2}{*}{ Results } & & \multicolumn{3}{|c|}{ Baghlani et al. [3] } & \multicolumn{2}{c|}{ Wu et al. [29] } \\
\cline { 2 - 6 } & $\mathrm{PSO}^{2}$ (Li et al.) & FA $^{3}$ & AFA $^{4}$ & FA $^{3}$ & IFA $^{5}$ \\
\hline Weight $(\mathrm{kg})$ & 285.35 & 247.32 & 247.09 & 256.91 & 256.91 \\
\hline NOA $^{1}$ & 150,000 & 38,775 & 6,750 & 1,245 & 570 \\
\hline
\end{tabular}




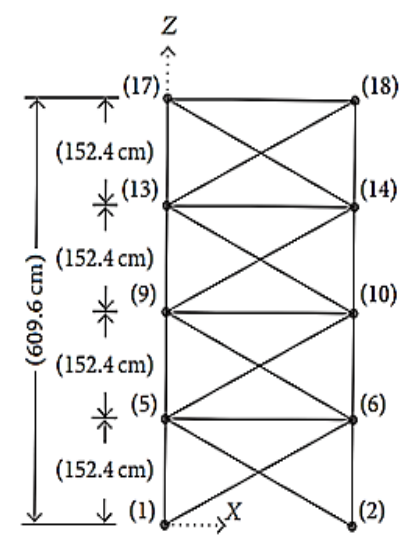

Fig. 3(a): 72-bar spatial truss

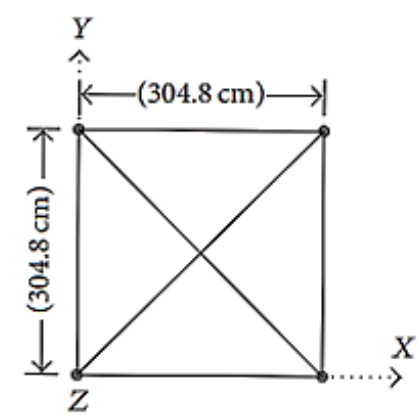

Fig. 3(b): Planner view numbering

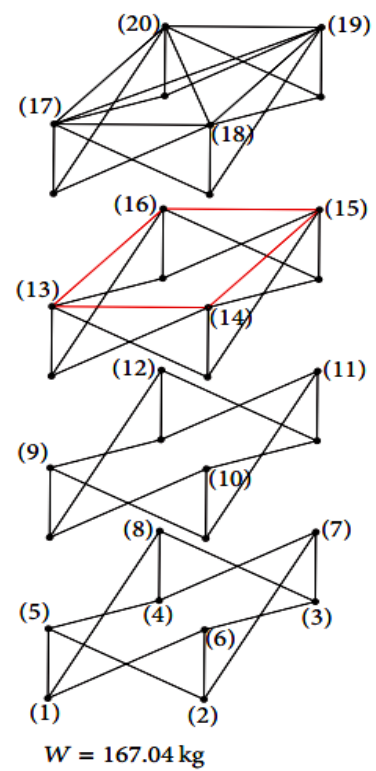

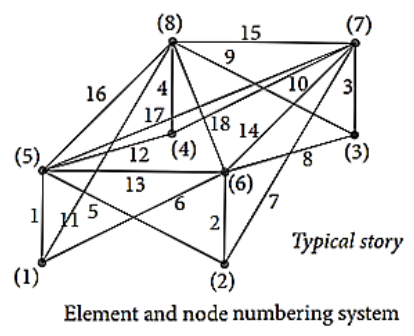

Fig. 3(c): Element \& node

Fig. 3(d): Best topology of 25-bar spatial truss

Table 2: Results for 72-bar spatial truss structure [29].

\begin{tabular}{cccccc}
\hline \multirow{2}{*}{ Results } & \multicolumn{3}{c}{ Baghlani et al [3] } & \multicolumn{2}{c}{ Wu et al. [29] } \\
\cline { 2 - 5 } & PSO $^{2}$ (Li et al.) & FA $^{3}$ & AFA $^{4}$ & FA $^{3}$ & IFA $^{5}$ \\
\hline Weight $(\mathrm{kg})$ & 2457.12 & 165.10 & 165.04 & 167.04 & 167.04 \\
NOA $^{1}$ & 150,000 & 41,085 & 11,000 & 14,280 & 9,520 \\
\hline
\end{tabular}

${ }^{1}$ Number of Analysis, ${ }^{2}$ Partical Swarm Optimization, ${ }^{3}$ Firefly Algorithm, ${ }^{4}$ Accelerated FA, Improved FA 
After the comparative study from table 1 and 2, Firefly Algorithm shows the best results than Particle Swarm Optimization (PSO). Firefly Algorithm seems effective in both i.e. solution quality as well as convergence speed. The result shows that the minimum weight obtained from the firefly algorithm is almost fifteen times lesser than it found from PSO. Similarly, the solutions of firefly algorithm converged nearly four times faster than PSO. Based on this result, it can be concluded that the modified versions of firefly algorithms (AFA and IFA) has the highest convergence speed and higher solution quality compared to another meta-heuristic method like PSO. It speaks clearly that the firefly algorithm must be the first selection when solving the complex structural engineering problems, like simultaneous optimization of size, shape, and topology a truss.

Similarly, if the results form firefly algorithm and modified versions of firefly algorithms (AFA and IFA) are compared, it can be pointed out that the modified versions of firefly algorithm remarkably speed up the convergence of solution than the speed of original version of firefly algorithm. That is almost four times faster in case of accelerated firefly algorithm and 1.5 times faster in case of improved firefly algorithm. From these results an important conclusion can make, that is, the solution obtained from the classical firefly algorithm is better than other meta-heuristic methods, but it may not be a final and sufficient one. Therefore, some proper control of its parameter and problem-based modifications are necessary to enhance the capacity of the classical algorithm. Ultimately, that helps to push the fireflies towards the best solution with the fastest convergence rate.

\section{Conclusion}

Firefly algorithm is becoming an important and powerful method in comparison to several other meta-heuristic methods, such as GA[13], PSO [23], HS [18], SA [5], Ant Colony Optimization [10] etc., because of its simplicity, versatility, superior efficiency. It has more flexibility to tread-off between exploration and exploitation. It means that the firefly algorithm is strong to search globally as well as local search in a design domain. Because of this property, the firefly algorithm can reach more near to the exact solution. Structural engineering is one of a complex field in engineering sector due to its challenges to implementing the original concepts into a real-world, economically and safely. Firefly algorithm will provide a better way to solve such problems efficiently. In these days, firefly algorithm becomes more popular in structural optimization field because of its robustness and flexibility to solve the various type of problems, such as structural designs, structural optimization, structural damage detection, earthquake resistance design, earthquake prediction etc. including NP-hard problems. From the literature study, it can be concluded that; firefly algorithm is a very strong method than other meta-heuristic methods, to solve the complex practical engineering problems. Although firefly algorithm is stronger than other, still its solution may not be a final and sufficient. It demands problem-based modification if the research focused to find the best result in the fastest way.

Research community of structural engineering is struggling to find a best and efficient algorithm. From continuous study and its advancement in future, the firefly algorithm may change into a truly intelligent algorithm. Firefly algorithm has no long history in structural optimization field. There is a sufficient gap for future research in this area. Some of them are; (1) Firefly algorithm is rarely used to solve the problem with discrete design variables. The improvement of the algorithm for this aspect may be one of a probable area of future research. (2) Firefly algorithm has not the provision to change its parameter when Firefly moves towards the best solution. It may lead 
to reducing the strength of the firefly algorithm. Hence, to develop this method with parameter tuning and parameter controlling, may be one of a green area for future research. (3) There are some difficulties to apply the firefly algorithm in the structural engineering problems because of its limited theoretical study. Study about the theory of firefly algorithm with the objective of real-world application may be one another research area in future. (4) Almost negligible firefly Algorithm based research has carried out in structural earthquake engineering, therefore it may be a larger area in the future research.

\section{References}

[1] Abdullah A, Deris S, Mohamad MS and Hashim SZM (2012), A New Hybrid Firefly Algorithm for Complex and Nonlinear Problem, Advances in Intelligent and Soft Computing, 151: 673-680.

[2] Abshouri A and Meybodi M (2011), New Firefly Algorithm based on multi swarm and learning automata in dynamic environments, in: IEEE proceedings, 73-77.

[3] Baghlani A, Makiabadi MH and Rahnema H (2013), A new accelerated Firefly Algorithm for size optimization of truss structures, Scientia Iranica, 20(6): 1612-1625.

[4] Bendsoe M and Sigmund O (2002), Topology Optimization: Theory, Methods and Applications, Springer-Verlag, Berlin Heidelberg, New York.

[5] Bertsimas D and Tsitsiklis J (1993), Simulated annealing, Statistical Science, 8(1): 10-15.

[6] Blum C and Roli A (2003), Metaheuristics in combinatorial optimization: Overview and conceptual comparison, ACM Computing Surveys, 35(3): 268-308.

[7] Brajevic I and Ignjatovic J (2015), An Enhanced Firefly Algorithm for Mixed Variable Structural Optimization Problems, Ser. Math. Inform., 30(4): 401-417.

[8] Carbas S (2016), Design optimization of steel frames using an enhanced Firefly Algorithm, Engineering Optimization, 48(12): 2007-2025.

[9] Chai-Ead N, Aungkulanon P and Luangpaiboon P (2011), Bees and Firefly Algorithm for noisy non-linear optimization problems, in: Proceedings of the International Multi Conference of Engineering \& Computer Scientists, 2: 1449 -1454.

[10] Dorigo M and Caro GD (1999), The ant colony optimization meta-heuristic, McGraw Hill, London, UK.

[11] Erdal F (2017), A Firefly Algorithm for optimum design of new-generation beams, Engineering Optimization, 49(6): 915-931.

[12] Escobar CM, Estrada OAG and Acevedo HGS (2017), Damage detection in a unidimensional truss using the Firefly Algorithm and finite elements, Mecanica, A009.

[13] Farahani S, Abshouri A, Nasiri B and Meybodi M (2012), Some hybrid models to improve Firefly Algorithm performance, Int J Artif Intell. 8(12): 97-117.

[14] Fister I, Gandomi AH, Mousavi M and Farhadi A (2014), Soft Computing in Earthquake Engineering: A Short Overview, International Journal of Earthquake Engineering \& Hazard Mitigation, 2(2): 42-48.

[15] Fistera I, Iztok Fister I, Yang X and Bresta J (2013), A comprehensive review of Firefly Algorithm, Swarm and Evolutionary Computation, 13: 34-46.

[16] Gandomi AH, Yang XS and Alavi AH (2011), Mixed variable structural optimization using Firefly Algorithm, Computers \& Structures, 89: 23-24.

[17] Gomes HM (2012), A Firefly metaheuristic structural size and shape optimization with natural frequency constraints, International Journal of Metaheuristics, 2(1): 38-55. 
[18] Guo L, Wang G, Wang H and Wang D (2013), An Effective Hybrid Firefly Algorithm with Harmony Search for Global Numerical Optimization, The Scientific World Journal, Volume 2013, 1-9, http://dx.doi.org/10.1155/2013/125625

[19] Hashmi A, Goel N, Goel S and Gupta D (2013), Firefly Algorithm for Unconstrained Optimization, Journal of Computer Engineering, 11(1): 75-78.

[20] Horng M and Jiang T (2010), Multilevel image thresholding selection based on Firefly Algorithm, 7th International Conference on IEEE: 58-63.

[21] Husselmann A and Hawick K (2011), Parallel parametric optimization with Firefly Algorithms on graphical processing units, Technical Report, CSTN-141.

[22] Jati GK, Manurung R and Suyanto (2013), Discrete Firefly Algorithm for Traveling Salesman Problem: A New Movement Scheme, Swarm Intelligence and Bio-Inspired Computation: 295312.

[23] Kennedy J and Eberhart RC (1995), Swarm intelligence in cellular robotic systems, in: Proceedings of IEEE International Conference on Neural Networks, Piscataway, NJ: 1942 $-1948$.

[24] Miguel LFF, Lopez RH and Miguel LFF (2013), Multimodal size, shape, and topology optimization of truss structure the Firefly Algorithm, Advances in Engineering Software, 56: 23-37.

[25] Qingjie HU, Qingpeng LI, and Yue WU (2015), Discrete Firefly Algorithm in the application of topology optimization of Truss Structures, Proceedings of $11^{\text {th }}$ Asian Pacific Conference on Shell and Spatial Structures-APCS2015.

[26] Storn R and Price K (1997), Differential evolution simple and efficient heuristic for global optimization over continuous spaces, Journal of Global Optimization, 11(4): 341- 359.

[27] Talbi E (2009), Metaheuristics, Wiley \& Sons Inc., Hoboken, New Jersey.

[28] Tashkova K, Silc J, Atanasova N and Dzeroski S (2012), Parameter estimation in a nonlinear dynamic model of an aquatic ecosystem with meta-heuristic optimization, Ecological Modelling, 226: 36-61.

[29] Wu Y, Li Q, Hu Q and Borgart A (2017), Size and Topology Optimization for Trusses with Discrete Design Variables by Improved Firefly Algorithm, Mathematical Problems in Engineering, 2017: $1-12$.

[30] Yang X (2008), Nature-Inspired Metaheuristic Algorithms, $1^{\text {st }}$ ed., Luniver Press, BA11 6TT, UK.

[31] Yang X (2010), Nature-Inspired Metaheuristic Algorithms, $2^{\text {nd }}$ ed., Luniver Press, BA11 6TT, UK.

[32] Yang XS (2010), Firefly algorithm, levy flights and global optimization. in: Research and Development in Intelligent Systems.

[33] Yang XS (2011), Metaheuristic optimization: algorithm analysis and open problems, in: $P$. Pardalos, S. Rebennack (Eds.), Experimental Algorithms, Lecture notes in computer science, 6630: 21-32.

[34] Yang XS (2012), Multi-objective Firefly Algorithm for Continuous Optimization, Engineering with Computers, 29(2): 175-184.

[35] Yang XS, Cui Z, Xiao R, Gandomi AH and Karamanoglu M (2013), Swarm Intelligence and Bio-Inspired Computation, Elsevier Inc., Jamestown, London. 